\title{
Ternary Zero Correlation Zone Sequence Sets for Asynchronous DS-CDMA
}

\author{
Benattou Fassi' ${ }^{1}$ Ali Djebbari' ${ }^{1}$ Abdelmalik Taleb-Ahmed² \\ ${ }^{1}$ Telecommunications and Digital Signal Processing Laboratory, Djillali Liabes University of Sidi Bel Abbes, \\ Sidi Bel Abbes, Algeria \\ ${ }^{2}$ LAMIH UMR CNRS 8530, University of Valenciennes and Hainaut-Cambresis (UVHC), le Mont Houy, \\ France \\ Email: fassibenattou@yahoo.fr, adjebari2002@yahoo.fr, abdelmalik.taleb-ahmed@univ-valenciennes.fr
}

Received 10 August 2014; revised 10 September 2014; accepted 8 October 2014

Copyright (C) 2014 by authors and Scientific Research Publishing Inc.

This work is licensed under the Creative Commons Attribution International License (CC BY).

http://creativecommons.org/licenses/by/4.0/

(c) ()

\begin{abstract}
In this paper we propose a new class of ternary Zero Correlation Zone (ZCZ) sequence sets based on binary ZCZ sequence sets construction. It is shown that the proposed ternary ZCZ sequence sets can reach the upper bound on the ZCZ sequences. The performance of the proposed sequences set in asynchronous Direct Sequence-Code Division Multiple Access (DS-CDMA) system is evaluated. In the simulation we used two types of channels: Additive White Gaussian Noise (AWGN) and frequency non-selective fading with AWGN noise. The proposed ternary ZCZ sequence sets show better results, in term of Bit Error Rate (BER), than Hayashi's ternary ZCZ sequence sets.
\end{abstract}

\section{Keywords}

Hadamard Matrix, Zero Correlation Zone Sequences, Correlation, Asynchronous DS-CDMA, BER

\section{Introduction}

In Code Division Multiple Access (CDMA) systems, the number of spreading sequences determines the number of users and their correlation properties have a significant effect on anti-interference performance of the system [1]. Different types of codes used in communications systems have been studied in order to reduce Multiple Access Interference (MAI) [2] [3]. For an interference-free communication, spreading codes should have zero auto-correlation and zero cross-correlation functions at out-of-phase state. So, spreading sequences with good correlation properties can be used to improve the performance of CDMA systems [3]. One class of spreading sequences called Zero Correlation Zone (ZCZ) sequences possesses good correlation properties but only in specific zones called Zero Correlation Zone $\left(Z_{C Z}\right)$. There are several intensive studies of CDMA systems using ZCZ 
sequences sets [1] [3]-[6]. Various classes of ternary ZCZ sequences sets have been constructed [3] [4] and [7]-[13]. Ternary ZCZ sequences have the advantage over binary ZCZ sequences that is, for a given sequence length, the set has longer $Z_{\mathrm{CZ}}$ lengths and more sequences, and we may employ such hardware in binary ZCZ sequence sets system [3]. Any ternary ZCZ sequences set TZCZ $\left(L^{\prime}, M^{\prime}, Z_{\mathrm{CZ}}^{\prime}\right)$ could be characterized by the sequence length $L^{\prime}$, the number of sequences $M^{\prime}$ and the zero correlation zone length $Z_{\mathrm{CZ}}^{\prime}$. An optimal ZCZ set is the one that provides the maximum number of codes for a given $Z_{\mathrm{CZ}}^{\prime}$ and sequences lengths. The proposed ternary ZCZ sequences set with TZCZ $\left(L^{\prime}, M^{\prime}, Z_{\mathrm{CZ}}^{\prime}\right)$ is derived from a binary ZCZ sequence set with $\operatorname{BZCZ}\left(L, M, Z_{\mathrm{CZ}}\right)$. When compared with previous works on ternary ZCZ sequence sets [3] [4] and [7]-[13], our proposed ZCZ sequence set approaches optimality.

The remainder of the paper is organized as follows.

After a review of preliminary considerations in Section 2, the proposed design for sequence construction is explained in Section 3. Example of new ZCZ sequence sets are presented in Section 4. The properties of the proposed sequence sets are explained in Section 5. In Section 6, we consider the performance of the proposed ternary ZCZ sequence sets compared with those in [3] and [10] for the asynchronous DS-CDMA system in both AWGN and nonselective fading with AWGN noise channels. At the end, we draw the concluding remarks.

\section{Preliminaries}

\subsection{Definition 1} For a pair of sequences $X_{j}$ and $X_{v}$ of length $L$, the aperiodic correlation function (ACF) $\varphi_{\left(x_{j}, X_{v}\right)}(\tau)$ is defined
as follows [14] [15]:

$$
\varphi_{\left(X_{j}, X_{v}\right)}(\tau)= \begin{cases}\sum_{i=0}^{L-\tau-1} x_{j, i} x_{v,(i+\tau)}, & \text { if } 0 \leq \tau<L \\ \sum_{i=0}^{L+\tau-1} x_{j,(i-\tau)} X_{v, \tau}, & \text { if }-L<\tau<0 \\ 0, & \text { if }|\tau| \geq L\end{cases}
$$

The periodic correlation function (PCF) between $X_{j}$ and $X_{v}$ at a lag $\tau$ is determined by [15] [16]:

$$
\begin{gathered}
\forall \tau, \theta_{\left(X_{j}, X_{v}\right)}(\tau)=\varphi_{\left(X_{j}, X_{v}\right)}(\tau \bmod L)+\varphi_{\left(X_{j}, X_{v}\right)}((\tau \bmod L)-L) \\
\forall \tau \geq 0, \theta_{\left(X_{j}, X_{v}\right)}(\tau)=\sum_{i=0}^{L-1} x_{j, i} X_{v,(i+\tau) \bmod (L)} \text { and } \theta_{\left(X_{j}, X_{v}\right)}(-\tau)=\theta_{\left(X_{v}, X_{j}\right)}(\tau)
\end{gathered}
$$

\subsection{Definition 2}

A set of $M$ sequences $\left\{X_{j}\right\}_{j=0}^{M-1}=\left\{X_{0}, X_{1}, \cdots, X_{M-1}\right\}$ is called zero correlation zone sequence set if the periodic correlation functions satisfy $[\overline{\overline{1}} \mathrm{i} 5][16]$ :

$$
0<|\tau| \leq Z_{c z}, \theta_{\left(X_{j}, X_{j}\right)}(\tau)=0
$$

and

$$
(j \neq v), 0 \leq|\tau| \leq Z_{c z}, \theta_{\left(X_{j}, X_{v}\right)}(\tau)=0
$$

\section{Proposed Sequence Construction}

The construction procedure of the new ternary sequence sets is presented. The construction is accomplished across the following three steps:

Step 1: The $j^{\text {th }}$ row of the Hadamard matrix $H$ of order $n$ is indicated by $h_{j}=\left[h_{j, 0}, h_{j, 1}, \cdots, h_{j, n-1}\right]$. A set of $2 n$ sequences $d_{j}$, each of length $2 n$, is constructed as follows [17]:

For $0 \leq j<n$ 


$$
\begin{aligned}
& d_{j+0}=\left[-h_{j}, h_{j}\right] \\
& d_{j+1}=\left[h_{j}, h_{j}\right]
\end{aligned}
$$

Step 2: For the first stage $p=0$, and for a fixed integer value $n$, we can generate, based on the schema for sequence construction in [15], a series of sets $\left\{B_{j}\right\}_{j=0}^{2 n-1}$ and $\left\{T_{j}\right\}_{j=0}^{2 n-1}$ of $2 n$ sequences as follows:

Both sequences sets $\left\{B_{j}\right\}_{j=0}^{2 n-1}$ and $\left\{T_{j}\right\}_{j=0}^{2 n-1}$ are constructed from the sequences set $\left\{d_{j}\right\}_{j=0}^{2 n-1}$. A pair of sequences $B_{j+0}$ and $B_{j+1}$ of length $L=\left(2^{p+2} n\right)$ are constructed by applying the interleaving operation of a sequence pair $\left(d_{j+0}, d_{j+1}\right)$ in Equations (6) and (7) [17] and a pair of sequences $T_{j+0}$ and $T_{j+1}$ are constructed by the interleaving operation of a sequence pair $d_{j+0}, d_{j+1}$ and from padding $Z$, which are zeros of length $K$, as follows:

For $0 \leq j<n$,

$$
\begin{gathered}
B_{j+0}=\left[d_{j+0,0}, d_{j+1,0}, d_{j+0,1}, d_{j+1,1}, \cdots, d_{j+0,2 n-1}, d_{j+1,2 n-1}\right] \\
B_{j+1}=\left[d_{j+0,0},-d_{j+1,0}, d_{j+0,1},-d_{j+1,1}, \cdots, d_{j+0,2 n-1},-d_{j+1,2 n-1}\right] \\
T_{j+0}=\left[d_{j+0,0}, Z, d_{j+1,0}, Z, d_{j+0,1}, Z, d_{j+1,1}, \cdots, d_{j+0,2 n-1}, Z, d_{j+1,2 n-1}, Z\right] \\
T_{j+1}=\left[d_{j+0,0}, Z,-d_{j+1,0}, Z, d_{j+0,1}, Z,-d_{j+1,1}, \cdots, d_{j+0,2 n-1}, Z,-d_{j+1,2 n-1}, Z\right]
\end{gathered}
$$

The length of a pair sequences $T_{j+0}$ and $T_{j+1}$ in Equations (10) and (11) is $L^{\prime}=\left(2^{p+2} n+2^{p+K+1} n\right)$ and the member size of a pair sequence sets $\left\{T_{j}\right\}$ and $\left\{B_{j}\right\}$ is $M=M^{\prime}=2 n$.

Step 3: For $p>0$, we may recursively build a new series of sets $\left\{B_{j}\right\}_{j=0}^{2 n-1}$ and $\left\{T_{j}\right\}_{j=0}^{2 n-1}$ by interleaving actual sets $\left\{B_{j}\right\}_{j=0}^{2 n-1}$ and $\left\{T_{j}\right\}_{j=0}^{2 n-1}$ respectively. Sets $\left\{B_{j}\right\}_{j=0}^{2 n-1}$ and $\left\{T_{j}\right\}_{j=0}^{2 n-1}$ are generated as follows:

For $0 \leq j<n$,

$$
\begin{gathered}
B_{j+0}=\left[B_{j+0,0}, B_{j+1,0}, B_{j+0,1}, B_{j+1,1}, \cdots, B_{j+0,4 n-1}, B_{j+1,4 n-1}\right] \\
B_{j+1}=\left[B_{j+0,0},-B_{j+1,0}, B_{j+0,1},-B_{j+1,1}, \cdots, B_{j+0,4 n-1},-B_{j+1,4 n-1}\right] \\
T_{j+0}=\left[T_{j+0,0}, T_{j+1,0}, T_{j+0,1}, T_{j+1,1}, \cdots, T_{j+0,(4 n(1+K))-1}, T_{j+1,(4 n(1+K))-1}\right] \\
T_{j+1}=\left[T_{j+0,0},-T_{j+1,0}, T_{j+0,1},-T_{j+1,1}, \cdots, T_{j+0,(4 n(1+K))-1},-T_{j+1,(4 n(1+K))-1}\right]
\end{gathered}
$$

The length of both sequences $B_{j+0}$ and $B_{j+1}$ in Equations (12) and (13) is equal to $\left(2^{p+2} n\right)$ [17] and the length of both sequences $T_{j+0}$ and $T_{j+1}$ is equal to $\left(2^{p+2} n+2^{p+1+K} n\right)$.

\section{Example of Construction}

1) For $n=2$ and $p=2$, the $\left\{B_{j}\right\}_{j=0}^{3}$ is generated as follows [17]:

$$
\begin{gathered}
B_{0+0}=[-1,-1,-1,1,1,1,-1,1,-1,-1,-1,1,1,1,-1,1,1,1,1,-1,1,1,-1,1,1,1,1,-1,1,1,-1,1] \\
B_{1+0}=[-1,-1,-1,1,1,1,-1,1,1,1,1,-1,-1,-1,1,-1,1,1,1,-1,1,1,-1,1,-1,-1,-1,1,-1,-1,1,-1] \\
B_{0+1}=[-1,1,-1,-1,1,-1,-1,-1,-1,1,-1,-1,1,-1,-1,-1,1,-1,1,1,1,-1,-1,-1,1,-1,1,1,1,-1,-1,-1] \\
B_{1+1}=[-1,1,-1,-1,1,-1,-1,-1,1,-1,1,1,-1,1,1,1,1,-1,1,1,1,-1,-1,-1,-1,1,-1,-1,-1,1,1,1]
\end{gathered}
$$

Figure 1 shows the periodic auto-correlation function (PACF) $(\forall j, j=v)$ given in Equation (3) of $B_{0+0}$, and Figure 2 shows the periodic cross-correlation function (PCCF) $(\forall j, j \neq v)$ given in Equation (3) of $B_{0+0}$ with $B_{1+0}$.

The PACF and PCCF confirm that $\left\{B_{j}\right\}$ is a ZCZ $(32,4,4)$ sequence set.

2) For $n=2$ and $p=2$, the proposed $\left\{T_{j}\right\}_{j=0}^{3}$ is generated as follows: 


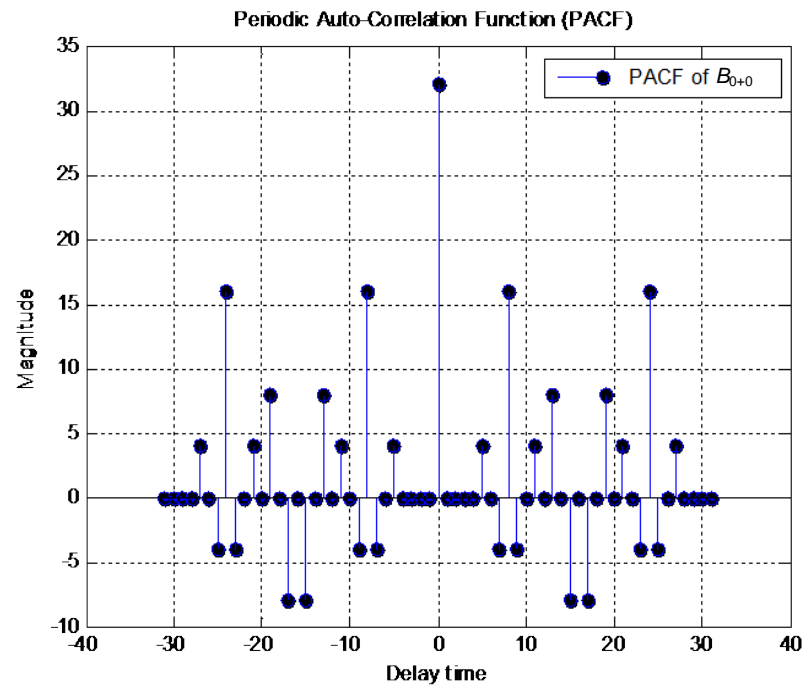

Figure 1. PACF of $B_{0+0}$.

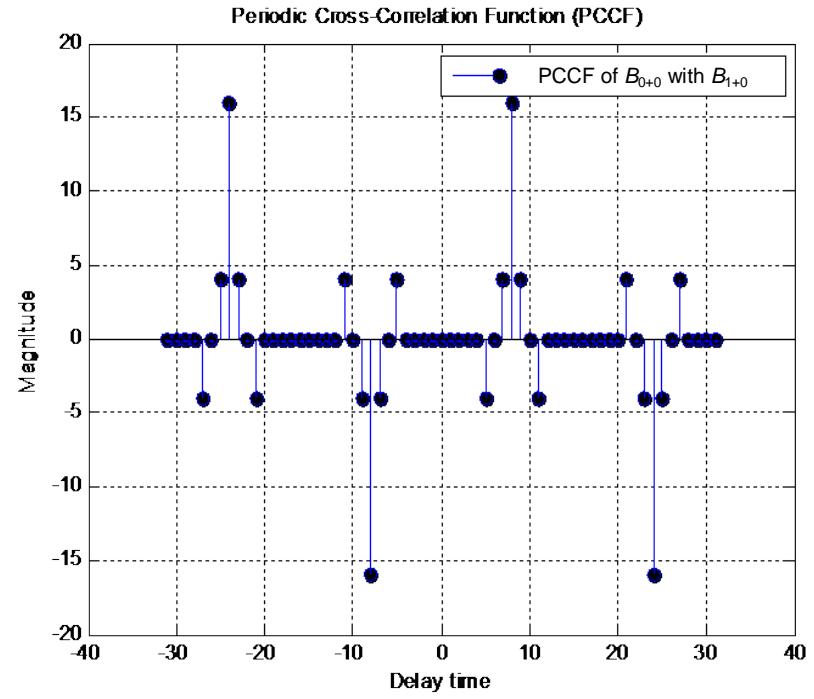

Figure 2. PCCF of $B_{0+0}$ with $B_{1+0}$.

$$
\begin{gathered}
T_{0+0}=\left[\begin{array}{r}
-1,-1,-1,1,0,0,0,0,1,1,-1,1,0,0,0,0,-1,-1,-1,1,0,0,0,0,1,1,-1,1, \\
0,0,0,0,1,1,1,-1,0,0,0,0,1,1,-1,1,0,0,0,0,1,1,1,-1,0,0,0,0,1,1,-1,1,0,0,0,0
\end{array}\right] \\
T_{1+0}=\left[\begin{array}{l}
-1,-1,-1,1,0,0,0,0,1,1,-1,1,0,0,0,0,1,1,1,-1,0,0,0,0,-1,1,-1,1,0,0,0,0, \\
1,1,1,-1,0,0,0,0,1,1,-1,1,0,0,0,0,-1,-1,-1,1,0,0,0,0,-1,-1,1,-1,0,0,0,0
\end{array}\right] \\
T_{0+1}=\left[\begin{array}{l}
-1,1,-1,-1,0,0,0,0,1,-1,-1,-1,0,0,0,0,-1,1,-1,-1,0,0,0,0,1,-1,-1,-1,0, \\
0,0,0,1,-1,1,1,1,0,0,0,0,-1,-1,-1,1,0,0,0,0,-1,1,11,0,0,0,0,-1,-1,-1,0,0,0,0
\end{array}\right] \\
T_{1+1}=\left[\begin{array}{l}
-1,1,-1,-1,0,0,0,0,1,-1,-1,-1,0,0,0,0,1,-1,1,1,0,0,0,0,-1,1,1,1,0,0,0,0, \\
1,-1,1,1,0,0,0,0,1,-1,-1,-1,0,0,0,0,-1,1,-1,-1,0,0,0,0,-1,1,1,1,0,0,0,0
\end{array}\right]
\end{gathered}
$$

Figure 3 shows the PACF of $T_{0+0}$, and Figure 4 shows the PCCF of $T_{0+0}$ with $T_{1+0}$. The PACF and PCCF confirm that $\left\{T_{j}\right\}$ is a TZCZ $(64,4,12)$ sequences set. 


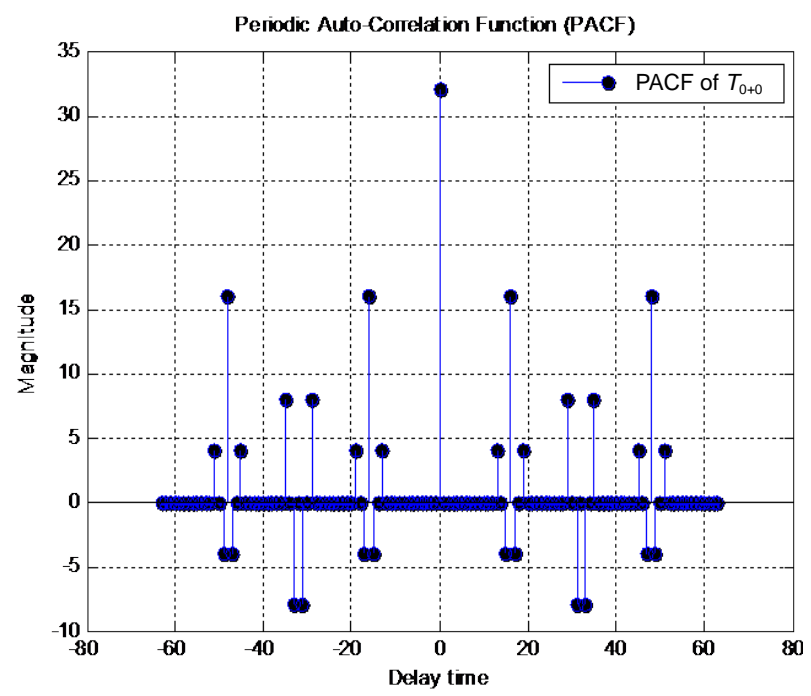

Figure 3. PACF of $T_{0+0}$.

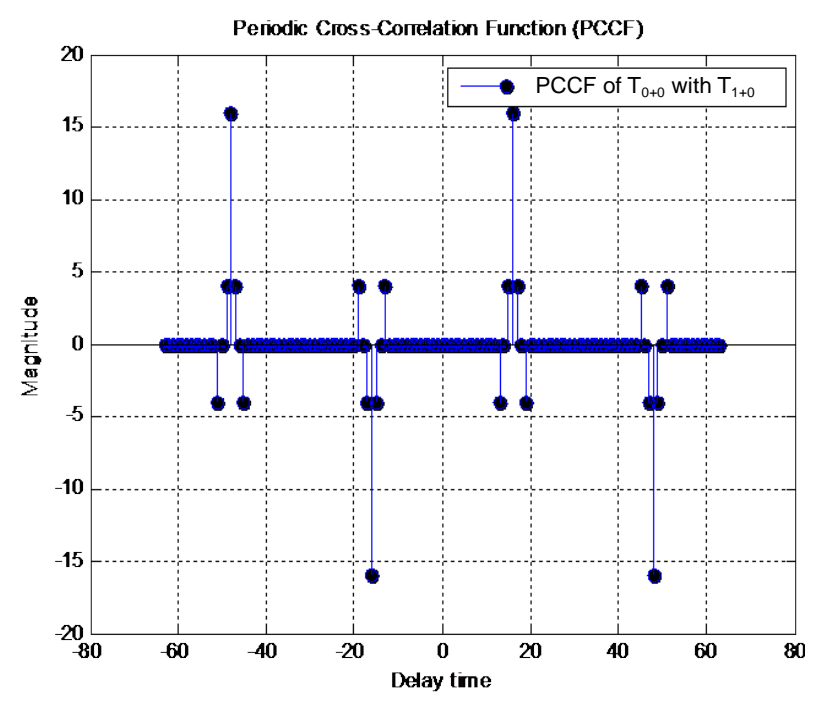

Figure 4. PCCF of $T_{0+0}$ with $T_{1+0}$.

\section{The Features of the Proposed Sequence}

The Binary ZCZ sequence set with BZCZ $\left(L, M, Z_{\mathrm{CZ}}\right)=\left(2^{p+2} n, 2 n, 2^{p}\right)$ is optimal or approach optimal binary ZCZ sequences [17]. The length of $T_{j}$ in Equations (14) and (15), equals $\left(L=2^{p+2} n+2^{p+1+K} n\right)$, is twice that of $T_{j}$ in Equations (10) and (11). Let $F=\left(2^{p+1}\right)$ and $Z_{p}=\left(2^{p+K}\right)$, the proposed ternary ZCZ sequence set with $\operatorname{TZCZ}\left(L^{\prime}, M^{\prime}, Z_{\mathrm{CZ}}^{\prime}\right)=\left(2^{p+2} n+2^{p+K+1} n, 2 n, 2^{p}+2^{p+K}\right)^{p}=\left(\mathrm{FM}+Z_{p} M, M, Z_{\mathrm{CZ}}+Z_{p}\right)$ is derived from the binary ZCZ sequence set BZCZ $\left(L, M, Z_{\mathrm{CZ}}\right)=\left(\mathrm{FM}, M, Z_{\mathrm{CZ}}\right)$. If the obtained ternary ZCZ sequence set is optimal, it satisfies the ratio $M^{\prime}=L^{\prime} /\left(Z_{\mathrm{CZ}}^{\prime}+1\right)$ [9] [10].

If $p=0$ (the first iteration), the obtained sequence set is an optimal ternary ZCZ sequence set.

Proof: Let $R=\frac{M^{\prime}\left(Z_{\mathrm{CZ}}^{\prime}+1\right)}{L^{\prime}}$, we calculate the following ratio:

$$
R=\frac{M^{\prime}\left(Z_{\mathrm{CZ}}^{\prime}+1\right)}{L^{\prime}}=\frac{2^{p}+2^{p+K}+1}{2^{p+1}+2^{p+K}}
$$

If $p=0, \forall K, R=1$. 
If $p \neq 0$ and the number $(K)$ of the padded zeros tends to the infinite, the obtained TZCZ sequences set (see Table 1 for $n=2$ ) is asymptotically optimal.

Proof: $R=\frac{M^{\prime}\left(Z_{\mathrm{CZ}}^{\prime}+1\right)}{L^{\prime}}=\frac{2^{K}+1}{2^{K}}$ and $\lim _{K \rightarrow \infty}(R)=1$

Noted that for this case, after spreading, the power of the symbol will decrease sharply, it is mandatory to compensate it, but this requirement increases Peak-to-Average Power Ratio (PAPR) and dynamic range of the transmitted signal [3].

From Table 2 we can see that the proposed sequence set in this paper can provide certain benefits. The length of sequences and $Z_{\mathrm{CZ}}$ will increase together while the number of sequences remains unchanged.

In the asynchronous DS-CDMA system, the time delay is typically in some chips, and for this, we can increase the $Z_{\mathrm{CZ}}$ to reduce MAI, but the member size will be relatively petty.

For a given member size $M$, we can find various sets of sequences with different lengths $L$ and $Z_{\mathrm{CZ}}$. As an example, assuming that $M=8$ and $K=3$ (see Table 3 for $n=4$ ), we can draw upper bounds of our code performance and compare it with the Hayashi's approach.

The Hayashi's ternary sequence sets ZCZ- $\left((n+1) 2^{p+2}, 2 n, 2^{p+1}-1\right)$ in [10] based on Hadamard matrix, the member size of the sequence set is $\frac{n}{n+1}$ of theoretical upper bound.

It is clear from Table 3, the proposed construction is one of the better type in the constructions mentioned in Table 2. Compared with the Hayashi's work, our proposed, in all cases, is optimal or approximate optimal ZCZ

Table 1 . The parameters of the proposed ternary ZCZ sequence set and the ratio $R$.

\begin{tabular}{cccccccccccccccc}
\hline$p$ & 0 & 0 & 0 & 0 & 0 & 1 & 1 & 1 & 1 & 1 & 2 & 2 & 2 & 2 & 2 \\
$K$ & 1 & 2 & 3 & 4 & 5 & 1 & 2 & 3 & 4 & 5 & 1 & 2 & 3 & 4 & 5 \\
$L^{\prime}$ & 16 & 24 & 40 & 72 & 136 & 32 & 48 & 80 & 144 & 272 & 64 & 96 & 160 & 288 & 544 \\
$Z_{\mathrm{Cz}}^{\prime}$ & 3 & 5 & 9 & 17 & 33 & 6 & 10 & 18 & 34 & 66 & 12 & 20 & 36 & 68 & 132 \\
$R$ & 1 & 1 & 1 & 1 & 1 & 0.88 & 0.92 & 0.95 & 0.97 & 0.99 & 0.81 & 0.88 & 0.93 & 0.96 & 0.98 \\
\hline
\end{tabular}

Table 2. Comparison of three types of ZCZ sequence sets.

\begin{tabular}{|c|c|c|c|c|c|}
\hline Constructed ZCZ sequence sets & $\begin{array}{l}\text { Length of } \\
\text { sequences }\end{array}$ & $\begin{array}{l}\text { Sequence } \\
\text { element }\end{array}$ & $\begin{array}{l}\text { Number } \\
\text { size }\end{array}$ & $Z_{\mathrm{cz}}$ & $\begin{array}{l}\text { Performance } \\
\text { parameter } R\end{array}$ \\
\hline $\mathrm{ZCZ}-\left(2^{p+2} n, 2 n, 2^{p}\right)$ in [17] & $2^{p+2} n$ & Binary & $2 n$ & $2^{p}$ & $\frac{\left(2^{p}+1\right)}{2^{p+1}}$ \\
\hline $\mathrm{ZCZ}-\left((n+1) 2^{p+2}, 2 n, 2^{p+1}-1\right)$ in $[10]$ & $2^{p+2}(n+1)$ & Ternary & $2 n$ & $2^{p+1}-1$ & $\frac{n}{n+1}$ \\
\hline $\mathrm{ZCZ}-\left(2^{p+2} n+2^{p+K+1} n, 2 n, 2^{p}+2^{p+K}\right)$ in this paper & $2^{p+2} n+2^{p+K+1} n$ & Ternary & $2 n$ & $2^{p}+2^{p+K}$ & $\frac{2^{p}+2^{p+K}+1}{2^{p+1}+2^{p+K}}$ \\
\hline
\end{tabular}

Table 3. The parameters of two constructions of ternary ZCZ sequence sets.

\begin{tabular}{|c|c|c|c|c|c|c|c|c|}
\hline \multirow{2}{*}{$\begin{array}{c}\text { Sequence sets } \\
L\end{array}$} & \multicolumn{4}{|c|}{ Hayashi's sequence set } & \multicolumn{4}{|c|}{ Proposed sequence set } \\
\hline & 80 & 160 & 320 & 640 & 80 & 160 & 320 & 640 \\
\hline$Z_{\mathrm{CZ}}$ & 7 & 15 & 31 & 63 & 9 & 18 & 36 & 72 \\
\hline$R$ & 0.8 & 0.8 & 0.8 & 0.8 & 1 & 0.950 & 0.925 & 0.912 \\
\hline
\end{tabular}


sequence set. Consequently it has a higher $Z_{\mathrm{CZ}}$ and better performance parameter $R$ than that given by Hayashi's construction.

\section{The Performance of the Asynchronous DS-CDMA System Using the Proposed Ternary ZCZ Sequence Set}

In this section, we consider the performance of the proposed ternary ZCZ sequence set used as spreading sequences for the DS-CDMA system shared by M asynchronous users simultaneously. In order to show this performance, the BER of an asynchronous DS-CDMA system over a frequency non-selective fading channel with AWGN noise estimated in [18] [19] is used:

$$
\mathrm{BER}=Q\left(\frac{T \sqrt{\frac{P}{2}}}{\sqrt{\frac{N_{0} T}{4}+\frac{T P^{2} \gamma^{2}}{4}+\left(1+\gamma^{2}\right) \sigma_{A}^{2}(i)}}\right)
$$

where $P$ is the common received power, $T$ is the symbol duration, the $Q$ function in Equation (16) is given by $Q(z)=\int_{z}^{\infty} \frac{1}{\sqrt{2 \pi}} \mathrm{e}^{\frac{-y^{2}}{2}} \mathrm{~d} y$ [20], the term $\left(\sigma_{n}^{2}=\frac{N_{0} T}{4}\right)$ is the variance for the AWGN noise, the term $\left(T P^{2} \gamma^{2} / 4\right)$ denote the faded component power from the user $i$ and $\sigma_{A}^{2}(i)$ is the global (non-faded) interference MAI power for the required $i$-th user.

Let $\gamma^{2}=0$, the BER of an asynchronous DS-CDMA system over AWGN channels is:

$$
\mathrm{BER}=Q\left(\frac{T \sqrt{\frac{P}{2}}}{\sqrt{\frac{N_{0} T}{4}+\sigma_{A}^{2}(i)}}\right)
$$

The MAI variance for the required $i$-th user can be calculated as [18] [19]

$$
\sigma_{A}^{2}(i)=\frac{T P^{2}}{12 L^{3}}\left(\sum_{\substack{m=1 \\ m \neq i}}^{M} r_{m, i}\right)
$$

where $r_{m, i}$ in Equation (18) is the interference term caused by all other users m except the user $i$. The term $r_{m, i}$ from Equation (1) can be written as [18] [19]:

Let $\varphi(\tau)=\varphi_{\left(X_{m}, X_{i}\right)}(\tau)$

$$
r_{m, i}=2 \sum_{\tau=1-L}^{L-1} \varphi(\tau)^{2}+\sum_{\tau=1-L}^{L-1} \varphi(\tau) \varphi(\tau+1)
$$

In Figure 5 and Figure 6 we compared the BER performance of the asynchronous DS-CDMA system employing Hayashi's TZCZ (40, 8, 3), Hayashi's TZCZ (20, 8, 1) and the proposed ternary ZCZ sequence sets with parameter TZCZ (32, 8, 3).

At BER $=10^{-4}$ in Figure 5, the system using constructed TZCZ can attain $01 \mathrm{~dB}$ and $06 \mathrm{~dB}$ gains over the same system employing Hayashi's TZCZ $(40,8,3)$ and Hayashi's TZCZ $(20,8,1)$ in AWGN respectively.

The BER performance, in Figure 6 was simulated assuming a frequency nonselective fading channel with AWGN noise with the common faded power ratio $\gamma^{2}=0.1$. As we can see in Figure 6, the proposed TZCZ sequence sets show better performance than Hayashi's TZCZ sequence sets.

At BER $=0.0015$ the system can attain $01 \mathrm{~dB}$ and $10 \mathrm{~dB}$ gains over the system employing Hayashi's TZCZ $(40,8,3)$ and Hayashi's TZCZ $(20,8,1)$ respectively. The amelioration over comparable Hayashi's ternary ZCZ sequences is due to the correlation properties of the proposed ternary sequence set.

\section{Conclusion}

A new construction method to create ternary ZCZ sequences set based on binary ZCZ sequence sets was proposed in this paper. This ternary ZCZ sequences set is either optimal or asymptotically optimal and their con- 


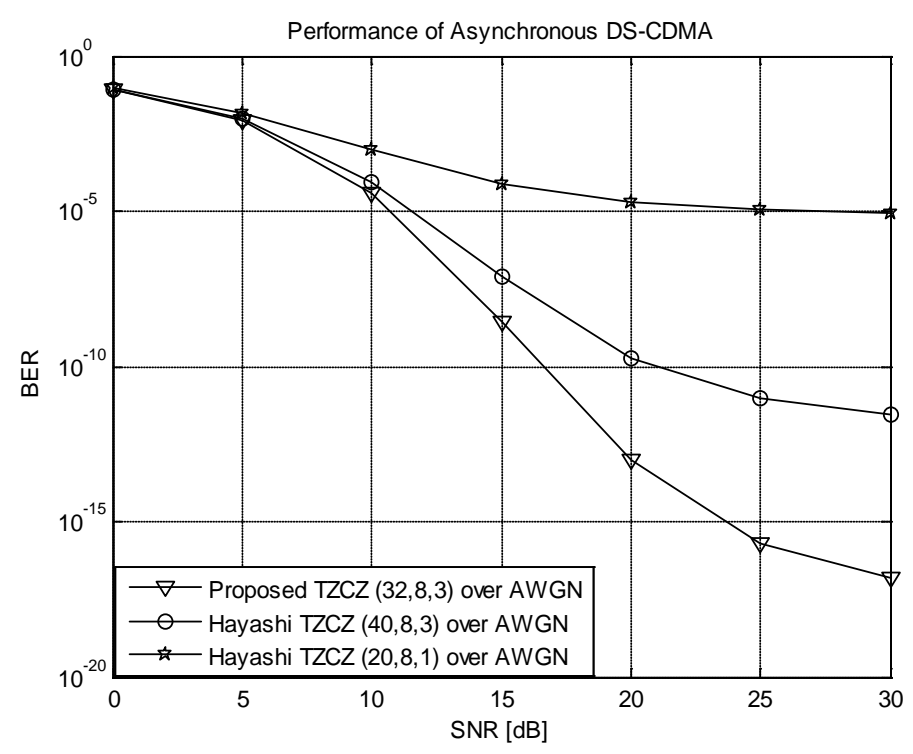

Figure 5. BER performance of Asynchronous DS-CDMA for different TZCZ over AWGN.

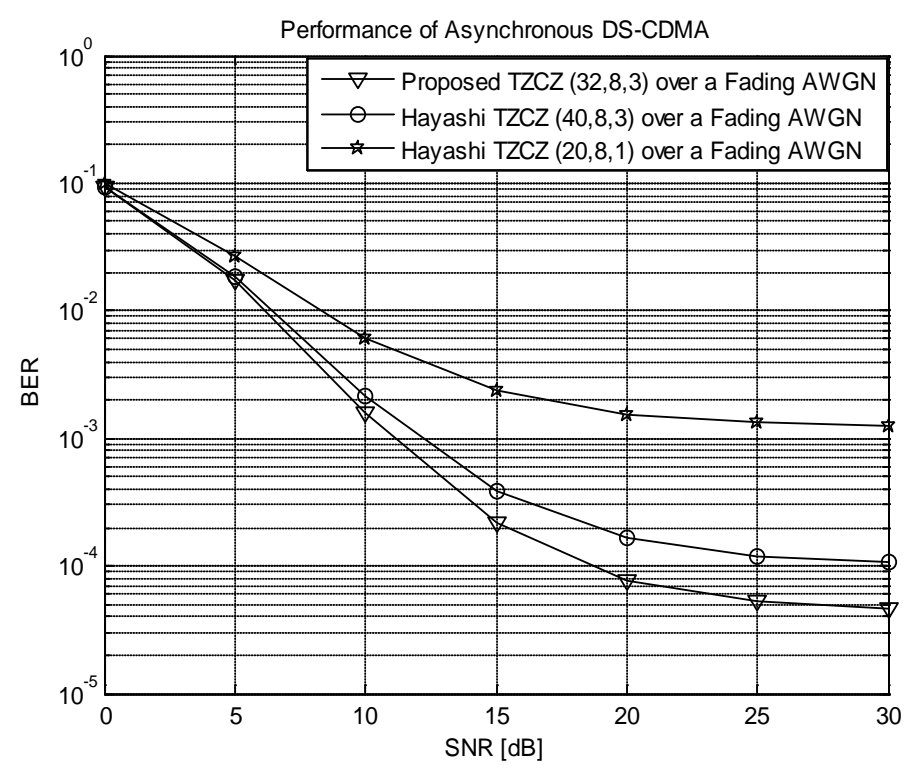

Figure 6. BER performance of Asynchronous DS-CDMA for different TZCZ over a Fading AWGN.

struction is more flexible than other ternary ZCZ constructions. The asynchronous DS-CDMA using the proposed ternary ZCZ sequences shows better BER performance in both AWGN and frequency non-selective fading channel with AWGN noise.

\section{References}

[1] Zhang, Z.Y., Ge, L.J., Yang, X.G., Zeng, F.X. and Xuan, G.X., AISS (2013) Construction of Multiple Mutually Orthogonal ZCZ Subsets for CDMA Communication Systems. 5, 695-704.

[2] Bai, Z.Q., Zhao, F., Wang, C.H. and Wang, C.-X., IJCS (2013) Multiple Access Interference and Multipath Interference Analysis of Orthogonal Complementary Code-Based Ultra-Wideband Systems over Multipath Channels. http://dx.doi.org/10.1002/dac.2623 
[3] Suk-Hoon, N. (2005) On ZCZ Sequences and Its Application to MC-DS-CDMA. Master of Science, Yonsei University, Seoul.

[4] Donelan, H. and O’Farrell, T. (2002) Large Families of Ternary Sequences with Aperiodic Zero Correlation Zones for a MC-DS-CDMA System. Proc. of 13th. IEEE Intl. SPIMRC, 5, 2322-2326.

[5] Karthikeyan, V. and Jeganathan Vijayalakshmi, V., IJEEE (2013) Analysis of Carrier Frequency Selective Offset Estimation-Using Zero-IF and ZCZ in MC-DS-CDMA. 1, 171-175.

[6] Huang, J.C., Matsufuji, S., Matsumoto, T. and Kuroyanagi, N., IJCS (2012) A ZCZ-CDMA System with BFSK Modulation. 25, 1620-1638.

[7] Wu, D., Spasojevié, P. and Seskar, I. Ternary Zero Correlation Zone Sequences for Multiple Code UWB. WINLAB, Rutgers University, 939-943.

[8] Wu, D., Spasojevié, P. and Seskar, I. (2003) Ternary Complementary Sets for Orthogonal Pulse Based UWB. WINLAB, Rutgers University, 1776-1780.

[9] Hayashi, T. and Matsufuj, S. (2006) On Optimal Construction of Two Classes of ZCZ Codes. IEICE TRANS, 89, 2345-2350. http://dx.doi.org/10.1093/ietfec/e89-a.9.2345

[10] Hayashi, T. (2003) A Class of Ternary Sequence Sets Having a Zero-Correlation Zone for Even and Odd Correlation Functions. Proc. IEEE ISIT, 434.

[11] Xu, S. and Li, D. (2003) Ternary Complementary Orthogonal Sequences with Zero Correlation Window. Proc. IEEE PIMRC, 49, 1669-1672.

[12] Takatsukasa, K., Matsufuji, S., Watanabe, Y., Kuroyanagi, N. and Suehiro, N. (2002) Ternary ZCZ Sequence Sets for Cellular CDMA System. IEICE TRANS, 85, 2135-2140.

[13] Cha, J.S., Electronics Letters (2001) Class of Ternary Spreading Sequences with Zero Correlation Duration. 37, 636637.

[14] Renghui, S., Xiaoqun, Z. and Li, L.Z. JCIT (2011) Research on Construction Method of ZCZ Sequence Pairs Set. 6, 15-23.

[15] Maeda, T., Kanemoto, S. and Hayashi, T. (2010) A Novel Class of Binary Zero-Correlation Zone Sequence Sets. Proc. IEEE TENCON, 708-711.

[16] Hayashi, T. (2009) A Class of Zero-Correlation Zone Sequence Set Using a Perfect Sequence. Signal Processing Letters, IEEE, 16, 331-334. http://dx.doi.org/10.1109/LSP.2009.2014115

[17] Fassi, B., Djebbari, A., Taleb-Ahmed, A. and Dayoub, I., IOSR-JECE (2013) A New Class of Binary Zero Correlation Zone Sequence Sets. 5, 15-19.

[18] Vladeanu, C. (2005) Optimum Chaotic Quantized Sequences for Asynchronous DS-CDMA System. University of Bucharest, Romania.

[19] Boulanger, C., Loubet, G., Lequepeys, J.R. and Ouvry, L. (1999) Direct Sequence Spread Spectrum Sequences. Traitement du Signal, 16, 426-436.

[20] Proakis, J.G. (2001) Digital Communications. 4th Edition, McGraw-Hill, New York. 
Scientific Research Publishing (SCIRP) is one of the largest Open Access journal publishers. It is currently publishing more than 200 open access, online, peer-reviewed journals covering a wide range of academic disciplines. SCIRP serves the worldwide academic communities and contributes to the progress and application of science with its publication.

Other selected journals from SCIRP are listed as below. Submit your manuscript to us via either submit@scirp.org or Online Submission Portal.
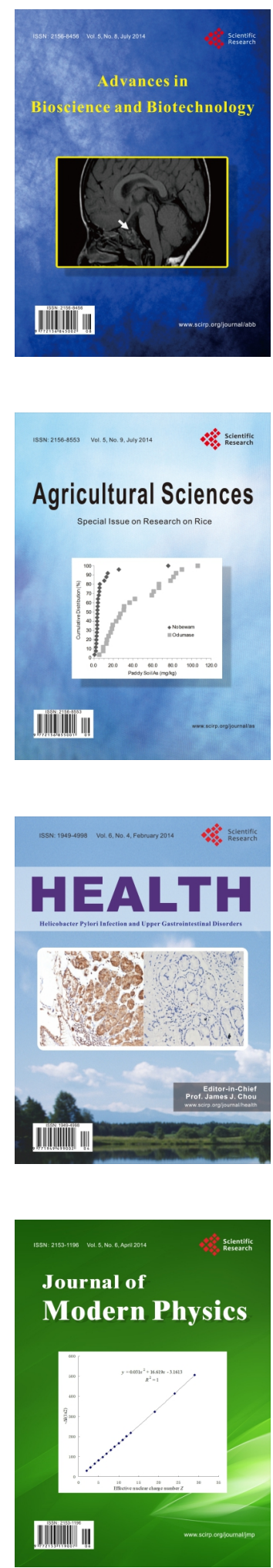
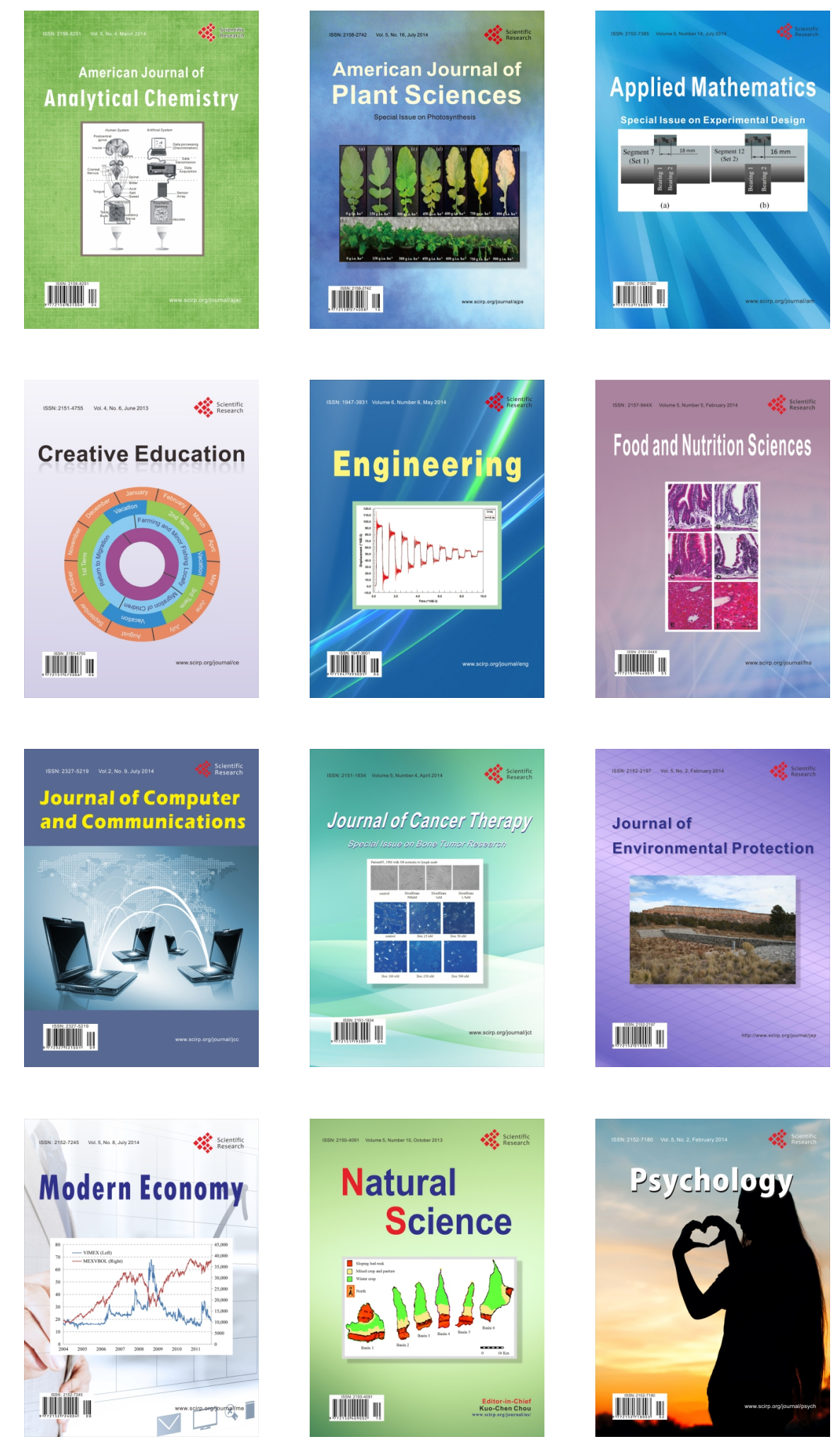